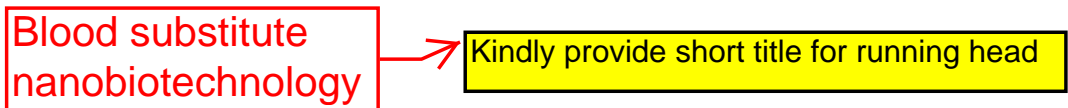

Nanobiotechnology-based Blood Substitutes and the Cardiovascular Systems

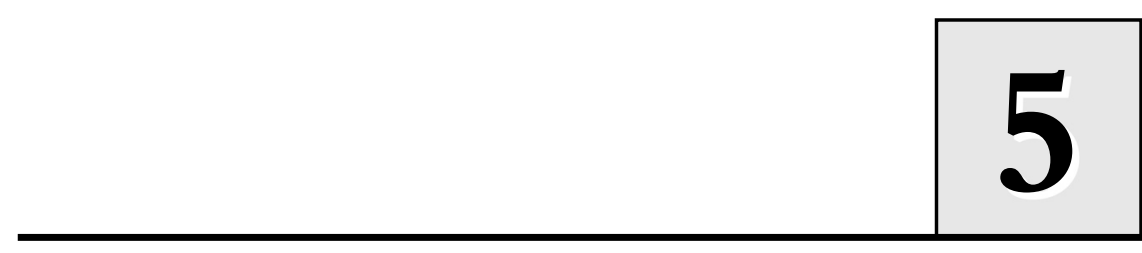

\title{
Nanobiotechnology-based Blood Substitutes and the Cardiovascular Systems in Transfusion Medicine
}

Thomas Ming Swi Chang

\begin{abstract}
Redblood cell membrane containsblood group antigens, and typing and matching are needed before donor blood can be transfused into patients. This results in delays in emergency situations. Donor blood requires storage at $4^{\circ} \mathrm{C}$ using standard methods and is good for less than $42 \mathrm{~d}$. Red blood cells cannot be sterilized to remove hepatitis viruses, HIV and other potential emerging infective agents. There is also a shortage of donor blood especially in emergencies, war or disasters. Nanobiotechnology has allowed the development of different types of red blood cell substitutes. The first-generation type is a simple modified hemoglobin oxygen carrier that has immediate potential for clinical uses. In particular, such substitutes do not have blood group antigens and can be
\end{abstract}

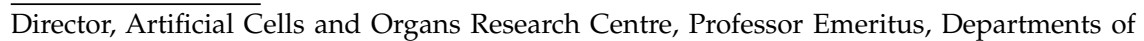
Physiology, Medicine and Biomedical Engineering, Faculty of Medicine, McGill University, 3655 Drummond Street, 10th floor, Room 1004, Montreal, Quebec, Canada H3G1Y6; E-mail: Artcell.med@mcgill.ca

List of abbreviations after the text. 
used immediately on the spot without matching or typing. Some can be stored for more than a year at room temperature. Infective agents such as HIV and other viruses and microorganisms can be removed. However, these first-generation red blood cell substitutes are only oxygen carriers and can have side effects in certain groups of patients and certain clinical conditions. New generations of red blood cell substitutes containing red blood cell antioxidant enzymes such as superoxide dismutase and catalase are being developed with promising results. The most advance red blood cell substitute is a nanodimension artificial red blood cell based on nanobiotechnology, but this is more complicated.

\section{INTRODUCTION}

Extracting the oxygen-carrying component, hemoglobin $(\mathrm{Hb})$, from red blood cell $(\mathrm{rbc})$ and purifying it to remove the membrane component would removed blood group antigens and also allow it to be sterilized. Unfortunately, $\mathrm{Hb}$ is a tetramer with two $\alpha$ subunits and two $\beta$ subunits $(\alpha 1 \beta 1 \alpha 2 \beta 2)$ (Perutz 1989). When free $\mathrm{Hb}$ is infused into the body, the tetramer $(\alpha 1 \beta 1 \alpha 2 \beta 2)$ breaks down into toxic dimers ( $\alpha 1 \beta 1$ and $\alpha 2 \beta 2)$, which causes renal toxicity and other adverse effects. The challenge lies in making use of the excellent oxygen-carrying properties of $\mathrm{Hb}$ while preventing its toxic effects.

Nanobiotechnology has been defined as the assembling of biological molecules into structures of 1 to $100 \mathrm{~nm}$ (in diameter of complex, thickness of membranes or diameter of nanotubules). The first nanobiotechnology approach reported in the literature is the use of bifunctional agent to crosslink hemoglobin into nanodimension thickness polyhemoglobin (PolyHb) membrane for artificial cell membrane (Chang 1964, 1972). This can also be used to assemble and crosslink $\mathrm{Hb}$ with bifunctional agent, using glutaraldehye, into soluble PolyHb of nanodimension (Chang 1971) (Fig. 1). This principle has been developed into different types of blood substitutes. First-generation rbc substitutes in the form of glutaraldehyecrosslinked hemoglobins are oxygen carriers for the replacement of the oxygen-carrying function of rbc (Gould et al. 2002; Jahr et al. 2008; Moore et al. 2009). One of these is in routine clinical use in South Africa (Jahr et al. 2008). There are also other types of first-generation Hb-based oxygen carriers (Fig. 1). Further developments are being made towards new generations of more complete blood substitutes. 


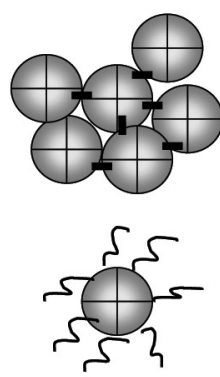

PolyHemoglobin

1964 Chang (Diacid)

1971 Chang (Glutaraldehyde)

Conjugated $\mathrm{Hb}$

1964 Chang (polyamide)

1968 Wong (dextran)

1970 Abuchowski \& Davis (PEGylation)

1980 Iwashita (polyethylene glycol)

Crosslinked tetrameric $\mathrm{Hb}$

1968 Bunn \& Jandl

1979 Walder et al (Diaspirin)

Recombinant Human $\mathrm{Hb}$

1990 Hoffman et al
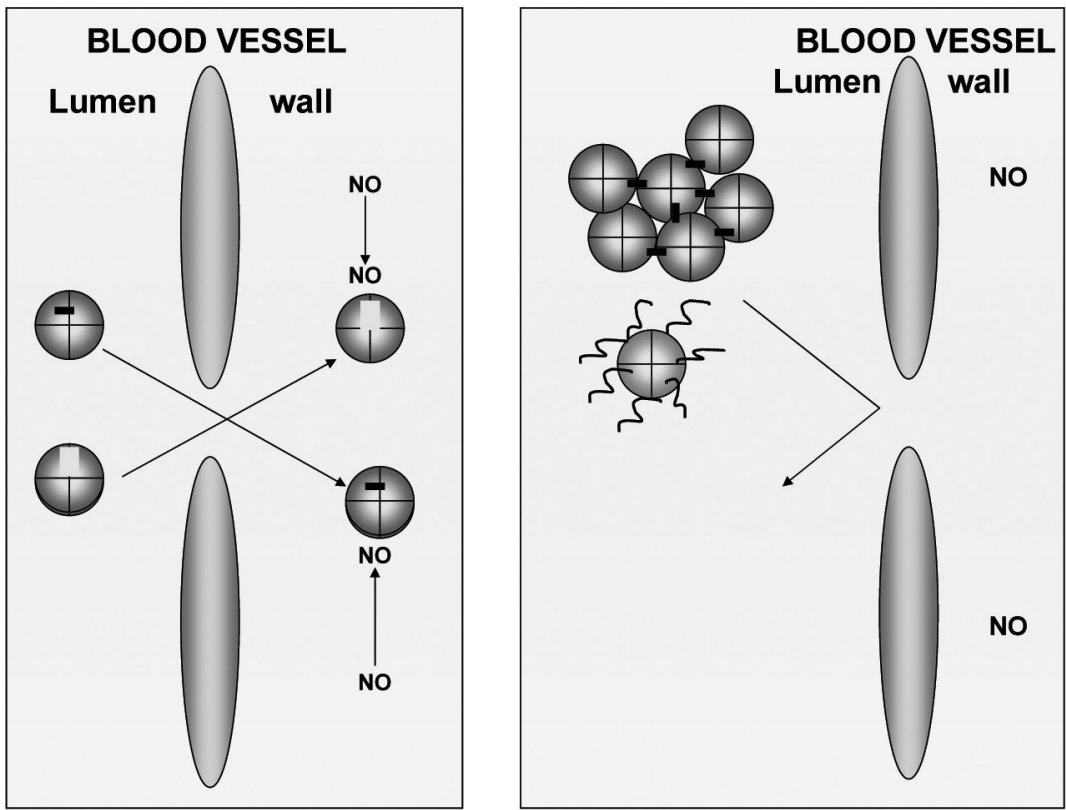

Fig. 1. Types of first-generation oxygen carriers as blood substitutes. Different types of firstgeneration blood carriers have been investigated and tested clinically. Single hemoglobin molecules resulted in much more vasopressor effects because they can cross the intercellular junction to bind and remove nitric oxide needed for normal vasoactivity (from Chang 2007, with permission). 


\section{FIRST-GENERATION OXYGEN CARRIERS}

Figure 1 summarizes the four general methods of $\mathrm{Hb}$ modification. These are $\mathrm{PolyHb}$ based on nanobiotechnology, conjugated $\mathrm{Hb}$, crosslinked tetrameric $\mathrm{Hb}$ and recombinant human $\mathrm{Hb}$. As seen from Fig. 1, most of the basic principles for modified $\mathrm{Hb}$ were available by the 1960s. Unfortunately, there was no public demand or interest until after 1986, when human immunodeficiency virus (HIV) in donor blood became a major public concern. However, last minute developments met with many setbacks because of lack of prior research and basic knowledge. The present status of first-generation oxygen carriers is as follows. $\leftarrow$
Polyhemoglobin is based on nanobiotechnology to assemble and crosslink the $\mathrm{Hb}$ molecules together into PolyHb. The first bifunctional agents used were sebacyl chloride (Chang 1964) and glutaraldehyde (Chang 1971). Later, two groups independently applied the glutaraldehydecrosslinked Poly $\mathrm{Hb}$ principle to develop oxygen carriers for clinical trials (Gould et al. 2002; Moore et al. 2009; Jahr et al. 2008). One of these has been approved for routine use in South Africa, where there is serious problem with HIV-contaminated donor blood (Jahr et al. 2008).

Conjugated $\mathrm{Hb}$ was first formed by the use of sebacyl chloride to crosslink diamine and $\mathrm{Hb}$ to form insoluble conjugated $\mathrm{Hb}$ (Chang 1964,1972) (Fig. 1). This has been extended to soluble conjugated $\mathrm{Hb}$ (Fig. 1) formed by linking one $\mathrm{Hb}$ molecule to soluble polymers. A maleimidepolyethylene glycol conjugated $\mathrm{Hb}$ was developed for clinical trial (Olofsson et al. 2006; Winslow 2006).

Intramolecularly crosslinked single tetrameric $\mathrm{Hb}$ was based on the use of a bifunctional cross-linker to crosslink the $\mathrm{Hb}$ molecules internally (intramolecular) (Fig. 1). This has been extensively developed and tested in clinical trials that showed vasopressor effect (Winslow 2006).

Recombinant human $\mathrm{Hb}$ is formed by the fusion of the two $\alpha$ subunits of each $\mathrm{Hb}$ molecule to prevent its breakdown into half molecule (dimers) (Fig. 1). A new recombinant human $\mathrm{Hb}$ has been prepared that does not bind nitric oxide thus obviating the problem with vasopressor effect (Winslow 2006). This is also a potential source of $\mathrm{Hb}$ for PolyHb and conjugated $\mathrm{Hb}$ and other future generation $\mathrm{Hb}$ based blood substitutes.

\section{Vasopressor Effects}

Intramolecularly crosslinked $\mathrm{Hb}$ and first-generation recombinant $\mathrm{Hb}$ blood substitutes contain $100 \%$ of molecular dimension modified $\mathrm{Hb}$. Infusion causes vasopressor effects and also increased smooth muscle contractions. With another type of PolyHb that contains 36\% molecular dimension modified $\mathrm{Hb}$, significant vasoactivity and increased smooth 
muscle contractions were also observed when using larger volumes. On the other hand, systemic hypertension has not been a problem in clinical trials using PolyHb that contained $<1 \%$ molecular dimension modified $\mathrm{Hb}$ even when large volumes were infused (Gould et al. 2002). This has led to the proposal that the intercellular junctions of the endothelial lining of vascular wall allow molecular dimension $\mathrm{Hb}$ to enter into the interstitial space (Chang 1997; Gould et al. 2002) (Fig. 1). There, Hb acts as a sink in binding and removing nitric oxide needed for maintaining the normal tone of smooth muscles. This results in the constriction of blood vessels and other smooth muscles.

\section{Design of Efficacy Animal Study for Hemorrhagic Shock}

Various experimental designs were used in the testing of blood substitutes for the resuscitation of hemorrhagic shock. Those based on bleeding 30-40\% of theblood volume are valid only for studying volume replacement, but this degree of blood loss does not require rbc replacement. Thus, this model is not sensitive enough for testing the efficacy for rbc substitutes in hemorrhagic shock. Testing the effectiveness of blood substitutes for both volume and rbc replacement requires a more severe model. For instance, using a modified Wigger's model with the removal of 2/3 of the total blood volume, we studied the effect of a single transfusion on the longterm survival of the animals (Chang 1997). Our results show that PolyHb blood substitutes are as effective as whole blood and more effective than 3 volume Ringers lactate, hypertonic-saline, dextran or albumin (Fig. 2).

\section{RATS BLED 2/3 BLOOD VOLUME SURVIVAL > 14 DAYS}

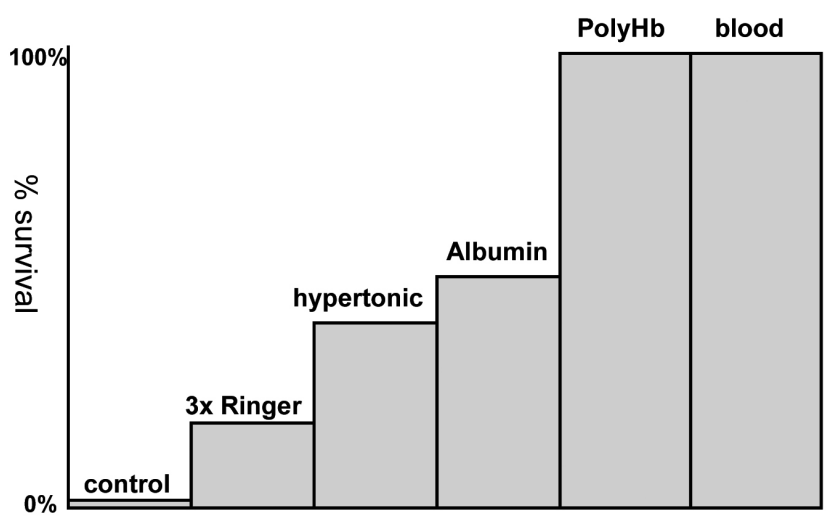

Fig. 2. Survival of hemorrhagic rat model. Long-term survival and recovery of $67 \%$ blood loss replaced with different types of solution. Only blood and PolyHb resulted in long-term survival (from Chang 2007, with permission). 


\section{Clinical Trials}

Gould et al. (2002) reported their clinical trials on 171 patients using glutaraldehyde-crosslinked human PolyHb with $<1 \%$ unpolymerized $\mathrm{Hb}$. They were able to replace extensive blood loss in trauma surgery and maintain the $\mathrm{Hb}$ level at the 8 to $10 \mathrm{~g} / \mathrm{dl}$ needed for safe surgery with no reported side effects. Infusion of PolyHb in patients with $\mathrm{Hb}$ level as low as $2 \mathrm{~g} / \mathrm{dl}$ can raise the $\mathrm{Hb}$ level to within the 8 to $10 \mathrm{~g} / \mathrm{dl}$ level with the patients recovering from surgery. Given that the supply of $\mathrm{Hb}$ from outdated donor blood is limited, a glutaraldehyde-crosslinked bovine PolyHb with $<3 \%$ unpolymerized molecular $\mathrm{Hb}$ has been developed and tested in clinical trials (Jahr et al. 2008). For example, the team has carried out multicenter, multinational, randomized, single-blind, rbc-controlled clinical trials in patients undergoing elective orthopedic surgery. A total of 688 patients are randomized 1:1 to receive either the $\mathrm{PolyHb}$ or rbc at the time of the first perioperative rbc transfusion decision: $59.4 \%$ of the patients receiving PolyHb required no rbc transfusion all the way to follow-up; $96.3 \%$ avoided transfusion with rbc on the first postoperative day; and up to $70.3 \%$ avoided rbc transfusion up to day 7 after. This bovine PolyHb has been approved for routine clinical use in patients in South Africa, a region with higher incidence of HIV.

A multicenter clinical trial on the use of polyhemoglobin in prehospital emergencies (Moore et al. 2009) showed that no typing or matching is needed and PolyHb can be used on the spot. This clinical trial involved 714 pre-hospital emergency patients (Moore et af. These 12009 were trauma patients with blood pressure of less than $90 \mathrm{mmHg}$ that were randomly divided into two groups: test group and control group. In this clinical trial, PolyHb was used on the spot in the ambulances, since, unlike donor blood, it required no typing or matching. The control group received standard management with saline infusion in the ambulance. The results showed that whereas the control group required rbc transfusion on admission into the hospital, the polyHb group required rbc transfusion only $14 \mathrm{~h}$ after admission into the hospital. However, there were side effects of myocardial infarction: $0.6 \%$ in the control group as compared to $3 \%$ in the PolyHb group. The authors of the study propose that PolyHb may be useful for patients at high risk of death when stored rbc are not available, even though there is some increase in frequency of adverse events (Moore et al.

This could be an important consideration in regions of the world with contaminated or insufficient donor blood. It could also be important in any emerging epidemic similar to HIV, or major civilian or armed conflicts. However, the FDA did not approve PolyHb for routine use in the United States, most likely because of the present availability of safe 
Reference to Table 1 not found $\checkmark$ added

Nanobiotechnology-based Blood Substitutes and the Cardiovascular Systems

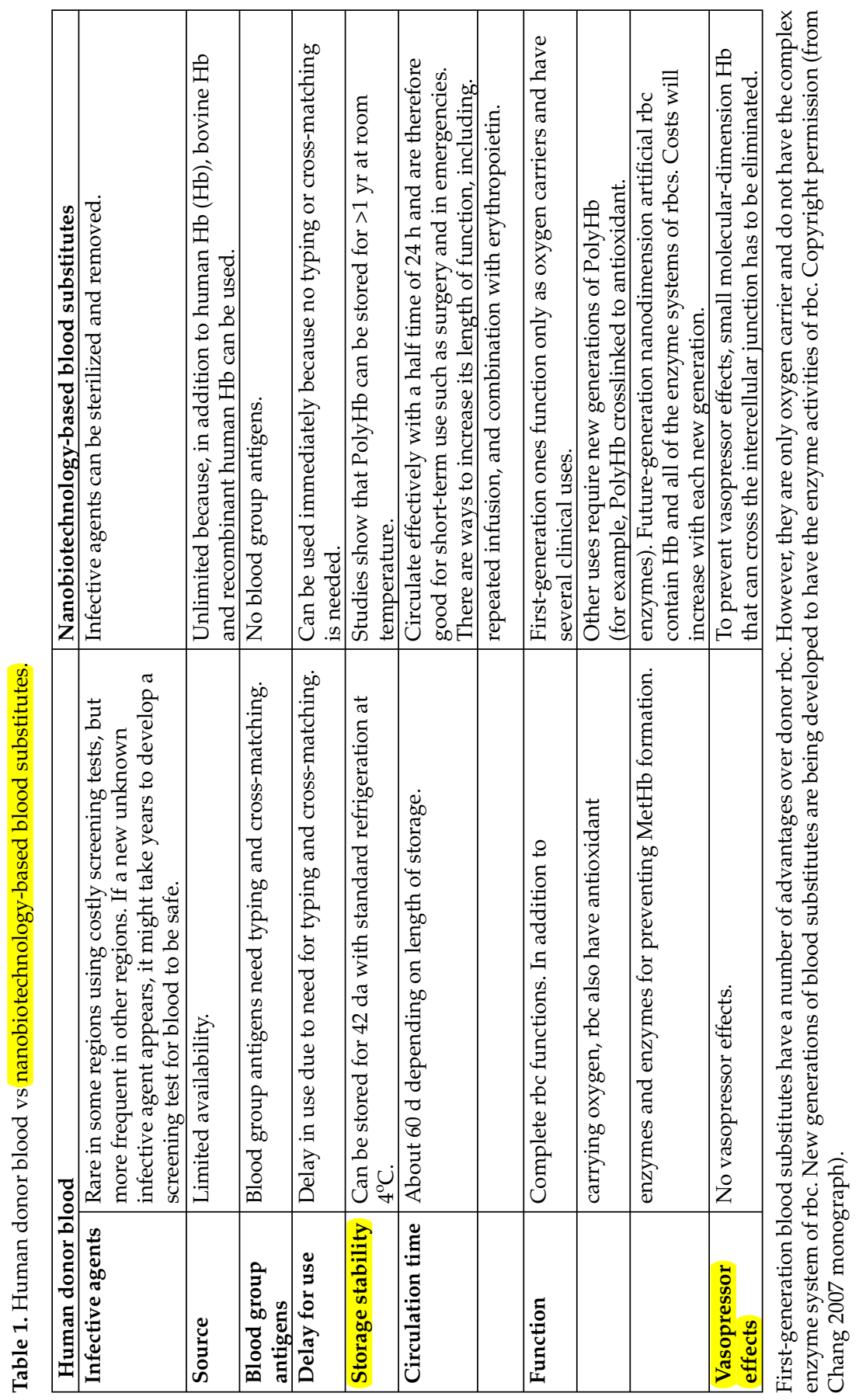


donor blood here. We also have to take regional and different situations into consideration. For example, in severe hemorrhagic shock with an $80 \%$ chance of death if there is not donor blood, what is the risk/benefit of PolyHb that would save lives (Fig. 2), but with a 3\% chance of side effects of myocardial infarction, as compared to $0.6 \%$ when using donor blood? In any case, we have to take all points of view into consideration and to carry out further research to gain the much needed basic information on the complicated topic of rbc substitutes (2010b). One important area is vasoactivity of blood substitutes.

\section{Vasoactivity}

As mentioned earlier, some of the earlier blood substitutes that are small tetrameric $\mathrm{Hb}$ or those with a large proportion of tetrameric $\mathrm{Hb}$ can result in vasoconstriction and hypertension. A controversial metaanalysis combined the clinical results of different types of $\mathrm{Hb}$, including intramolecularly crosslinked $\mathrm{Hb}$ that consists of $100 \%$ single molecules, and concluded that modified $\mathrm{Hb}$ causes vasoconstriction (Natanson et al. 2008). This meta-analysis did not take into consideration that modified $\mathrm{Hb}$ can be very different in composition and vasoactivity. On the other hand, we have to take all views into consideration and analyze all possibilities; further research is needed to clarify the issue of vasoactivity. Much research has been carried out on vasoactivity using different types of blood vessels, including hamster pouch microcirculation, aortic vessel ring perfusion, systemicblood pressure, and pulmonary artery perfusion. Since myocardial infarction is an important issue, we decided to test the effect of PolyHb on the coronary artery in rats. The heart rates in rats are very high and thus very sensitive to vasoconstriction, which results in a lack of oxygen supply. We prepared nanodimension PolyHb, each containing $0.4 \%, 16 \%$, $38 \%$ and $78 \%$ of tetramers using the same glutaraldehyde crosslinking and characterized to ensure that they all had the same oxygen affinity (Yu et al. 2006; Chang 2007). The results showed that PolyHb with $0.4 \%$ of tetramer did not cause ECG changes. With increasing percentage of molecular dimension modified $\mathrm{Hb}$, there is increasing elevation of the ST segment of the electrocardiogram. ST elevation is likely due to vasoconstriction of the coronary vessels resulting in decreased supply of oxygen to the heart. This result shows that vasoactivity varies extensively among different types of modified $\mathrm{Hb}$. Small hemoglobin molecules cross the intercellular junction to remove nitric oxide resulting in vasoconstriction (Fig. 1). Further support of the role of nitric oxide in vasopressor effects has come from study showing that inhalation of nitric oxide can prevent vasopressor effects from infusion of tetrameric hemoglobin (Yu et al. 2008). However, this is not as simple as just the tetrameric $\mathrm{Hb}$. Further studies (Yu et al. 
2010) show that although PolyHb with $<1 \%$ tetrameric $\mathrm{Hb}$ does not cause vasoconstriction in normal mice, it causes vasoconstriction in diabetic mice or mice fed with high lipid diet. This supports my proposal at the 2009 International Symposium on Blood Substitutes for the need to have more narrow indications and the exclusion of patients and conditions with arteriosclerosis and other endothelial dysfunctions especially in situations of oxidative stress (Chang, 2010b). There is a substantial proportion of patients in the 2009 clinical trial who are between the age of 45 to 78 and thus more likely to have problems related to arteriosclerosis (Moore 2009).

\section{SECOND-GENERATION RED BLOOD CELL SUBSTITUTES: OXYGEN CARRIERS WITH ANTIOXIDANT ACTIVITY}

\section{Ischemia-reperfusion}

In order to use blood substitutes for some clinical conditions, such as arteriosclerosis severe sustained hemorrhagic shock, or stroke, we have to consider the issue of oxygen radicals. In prolonged severe hemorrhagic shock, stroke, and other ischemic conditions, there is insufficient supply of oxygen resulting in ischemia. Ischemia stimulates the production of hypoxanthine at a rate proportional to the duration of ischemia. When the tissue is again perfused with oxygen, reperfusion, hypoxanthine is converted into superoxide. By several mechanisms, superoxide results in the formation of oxygen radicals that can cause tissue injury (Chang 1997; D'Agnillo and Chang 1998; Alayash et al. 2007). The enzymes in rbc help to prevent this to a limited extent. Thus, superoxide dismutase (SOD) in rbc converts superoxide into hydrogen peroxide that is in turn converted by catalase (CAT) into water and oxygen. However, in severe and prolonged ischemia, the normal amounts of these enzymes in the rbc are not enough to prevent ischemia-reperfusion injury. We have therefore used nanobiotechnology to prepare a soluble PolyHb-CAT-SOD complex with more CAT and SOD than those normally present in rbc (D'Agnillo and Chang 1998) (Fig. 3). This was done with a view to having an oxygen carrier with enhanced antioxidant enzymes to prevent ischemiareperfusion injuries.

\section{Oxygen Carrier with Antioxidant Activity}

In the form of PolyHb-CAT-SOD, the enzymes stay in the circulation together with PolyHb that has a half life of $24 \mathrm{~h}$ in human. This is unlike the rapid removal of free SOD and CAT from the circulation (10 and 20 $\mathrm{min}$, respectively). Furthermore, unless crosslinked to $\mathrm{Hb}$, these enzymes 

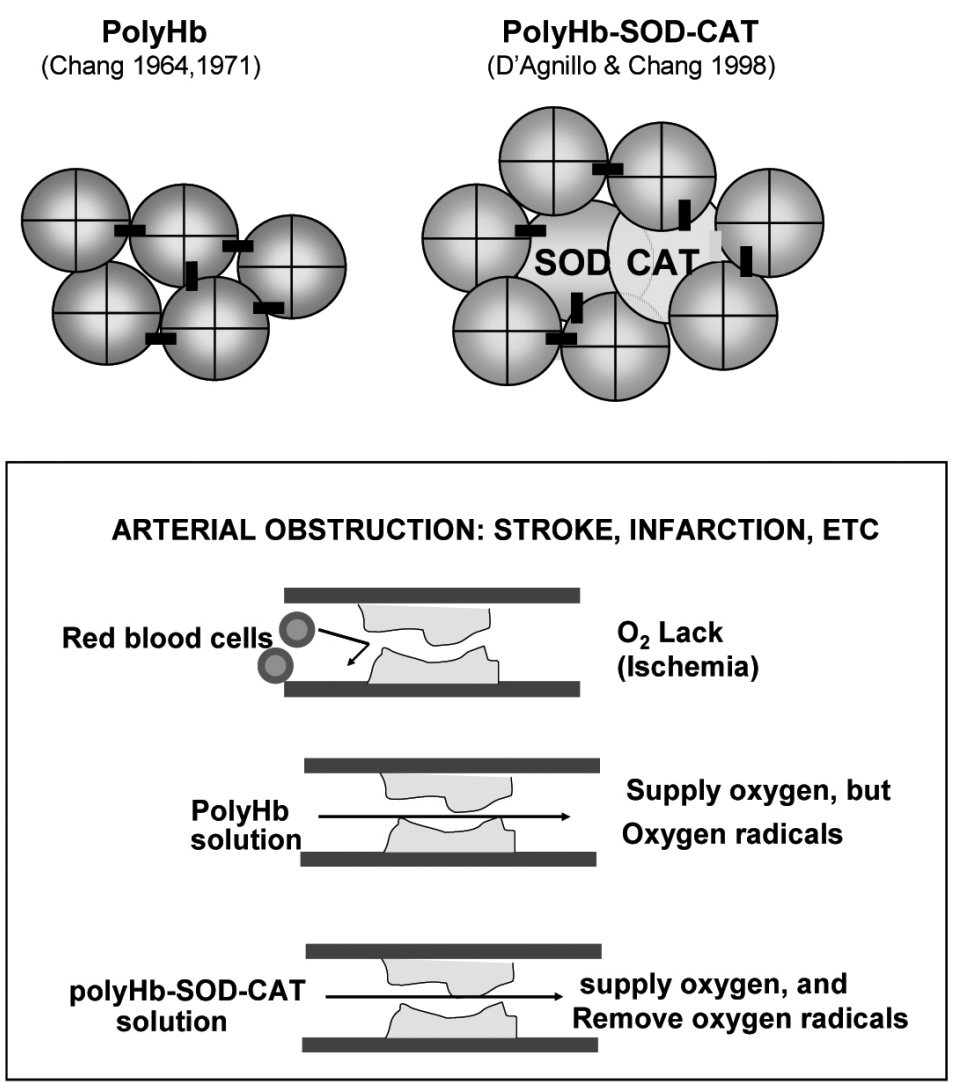

Fig. 3. Red blood cell and different types of blood substitutes in microcirculation. Arteriosclerosis causes narrowing and obstruction of blood vessels. Red blood cells, being 8 micron in diameter, have difficulty flowing through these partly obstructed vessels to supply the needed oxygen. PolyHb being a solution can perfuse partly obstructed vessels better than red blood cells to supply the needed oxygen. However, when ischemic tissue is perfused with an oxygen-rich solution, reactive oxygen species are generated, resulting in ischemia-reperfusion injury. Unlike PolyHb, PolyHb-SOD-CAT has the dual function of an oxygen carrier with the ability to remove oxygen radicals; like PolyHb, it is a solution and can perfuse partly obstructed vessels. (From Chang 2007, with permission.)

Color image of this figure appears in the color plate section at the end of the book.

are not located in close proximity to $\mathrm{Hb}$ and thus are less likely to give adequate protection to $\mathrm{Hb}$-initiated oxygen radicals. In vitro studies reveal that crosslinked $\mathrm{Hb}$-SOD-CAT is effective in removing oxygen radicals and hydrogen hydroxide, and in lessening damage to $\mathrm{Hb}$ and iron release. 


\section{Intestine and Ischemia-reperfusion}

In severe sustained hemorrhagic shock, reperfusion even with rbc that contain the normal amounts of CAT and SOD may not be enough to prevent ischemia-reperfusion. Since PolyHb-CAT-SOD can contain more enzymes than rbc, we tested this preparation for reperfusion in an ischemia-reperfusion intestine model (Chang 2007). The use of PolyHb for reperfusion results in a substantial increase in oxygen radicals. On the other hand, the use of PolyHb-CAT-SOD markedly and significantly decreased the formation of oxygen radicals

\section{The Brain and Ischemia-reperfusion}

Arteriosclerosis is the most common cause of narrowing and obstruction of blood vessels, resulting in myocardial infarction or stroke. Red blood cells, being 8 micron in diameter, have difficulty flowing through these partly obstructed vessels to supply the needed oxygen to the brain (Fig. 3). PolyHb, being a solution, can perfuse partly obstructed vessels better than rbc to supply the needed oxygen. However, when an ischemic heart or brain is perfused with an oxygen-rich solution, reactive oxygen species are generated, resulting in ischemia-reperfusion injury. PolyHb-SOD-CAT is a soluble complex and can perfuse the narrowed arteries. However, unlike PolyHb, PolyHb-SOD-CAT has dual function of an oxygen carrier with the ability to remove oxygen radicals (Fig. 3). Cerebral ischemia is also present in some cases of severe trauma, where there is hemorrhagic shock plus brain damage.

We used a combined hemorrhagic shock and transient global ischemia-reperfusion rat brain model to compare the use of PolyHb-SODCAT solution with four other oxygen-carrying solutions: (1) PolyHb, (2) PolyHb with CAT and CAT added as solution without crosslinking to PolyHb, (3) purified $\mathrm{Hb}$, and (4) oxygenated saline solution. Reperfusions were initiated $1 \mathrm{~h}$ after ischemia and the effects on blood-brain barrier and brain edema were followed for $6 \mathrm{~h}$. Cerebral edema was measured as changes in cerebral water content. There was no significant difference between the PolyHb-SOD-CAT and sham control group when followed for 2, 4 and $6 \mathrm{~h}$ (Powanda and Chang 2002) (On the other hand, significant increases in cerebral water content were observed after 4 h for PolyHb $(234.8 \pm 21.9 \mathrm{mg})$, free $\mathrm{Hb}$ plus SOD and CAT in free solution $(152.3 \pm 30.2 \mathrm{mg})$,oxygenated saline $(171.3 \pm 23.0 \mathrm{mg})$, and SF-Hb $(279.8 \pm$ $21.8 \mathrm{mg}$ ). Thus, except for PolyHb-CAT-CAT, all the solutions (oxygenated saline, $\mathrm{SF} \mathrm{Hb}$ and $\mathrm{PolyHb}$ ) resulted in cerebral edema. The result also shows that CAT and SOD have to be crosslinked into PolyHb-CAT-SOD to be effective in protecting against ischemia-reperfusion. 


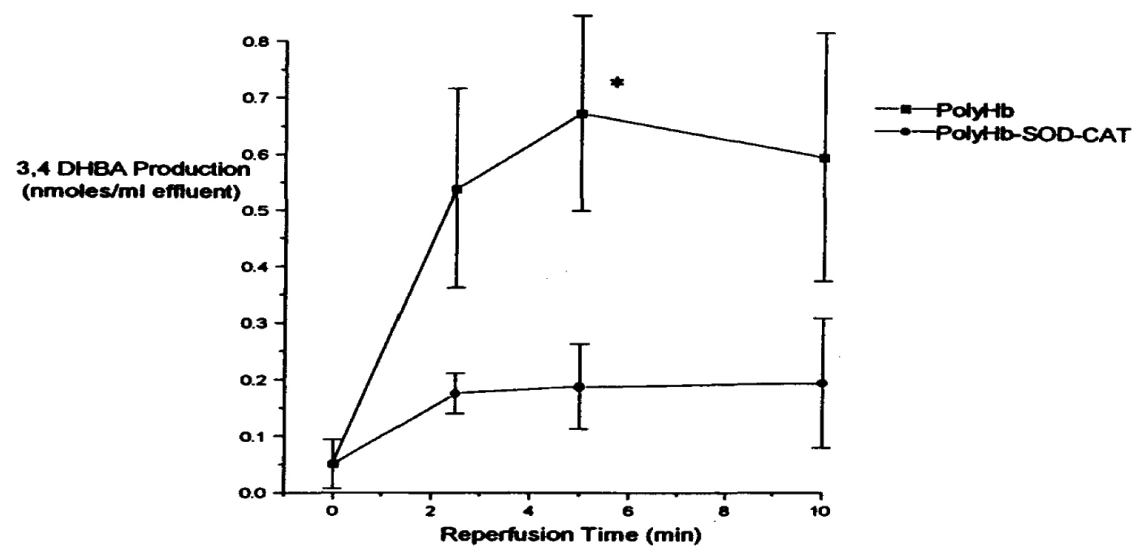

Fig. 4. Effects of perfusion of ischemic intestine on oxygen radicals. Oxygen radical measured as hydroxyl radical production assessed by the measurement of 3, 4 dihydroxybenzoate in ischemic intestine perfused with $5 \mathrm{~g} / \mathrm{dl}$ of PolyHb () or $5 \mathrm{~g} / \mathrm{dl}$ of PolyHb-SOD-CAT (). Intestinal effluent samples are collected during reperfusion at the indicated times. Data are presented as means \pm SD. * Statistical significance, $p<0.05$. (From Chang 2007, with permission.)

\section{Discussion}

The above studies show that in ischemia-reperfusion the use of PolyHbSOD-CAT resulted in significantly less production of oxygen radicals than PolyHb. This difference is much more obvious in the case of ischemiareperfusion of intestine or brain, both organs being particularly sensitive to ischemia-reperfusion injury. On the other hand, the hind limb is much less sensitive to ischemia-reperfusion injury even when using PolyHb. Another area of potential application that has been studied is use of PolyHb-SOD-CAT in preventing ischemia-reperfusion damage to sensitive donor organs such as the kidney or liver (Chang et al. 2004). Another area investigated is cardiopulmonary bypass. Ischemia-reperfusion injury has been noted to occur after cardiopulmonary bypass in children undergoing surgery for congenital heart disease (Shum-Tim). An extension of our PolyHb-SOD-CAT approach is the development of synthetic mimics of SOD for complexing with modified hemoglobin in the form of polynitroxyl hemoglobin with covalently bound nitroxides (Buehler et al. 2004). 


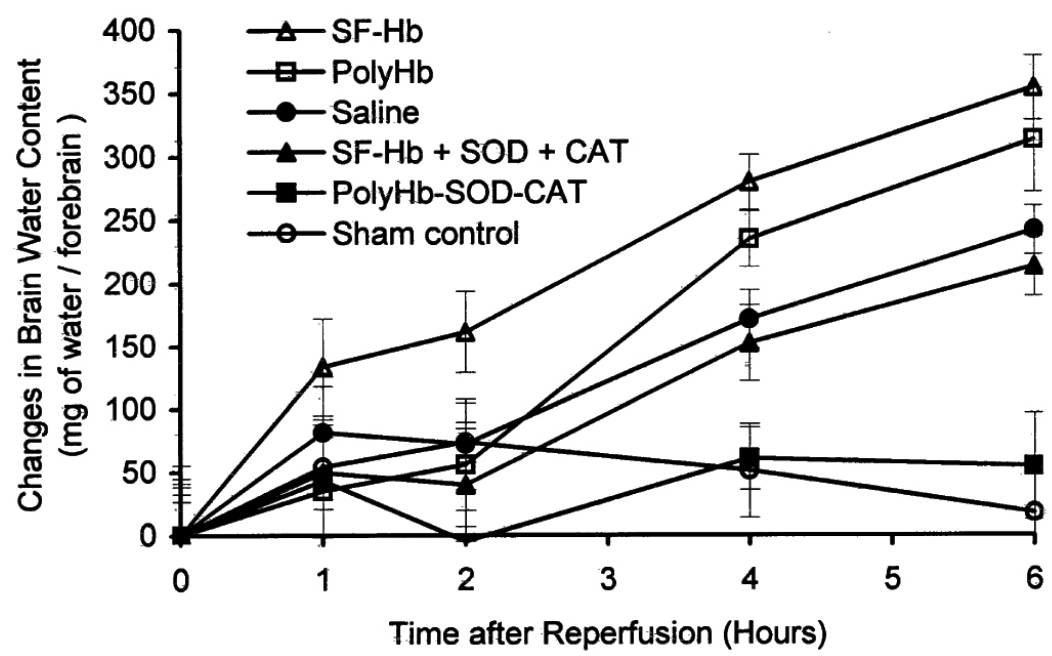

Reference to Fig. 5 not found

Fig. 5. Effects of reperfusion in ischemic brain on brain edema. This is followed as change in brain water content. The changes in brain water content of PolyHb-SOD-CAT treated animals are not significantly different from those of the sham control. The increase in water contents of saline, SF- $\mathrm{Hb}, \mathrm{SF}-\mathrm{Hb}+\mathrm{SOD}+\mathrm{CAT}$, and PolyHb are significantly different from that of the sham control and PolyHb-SOD-CAT group by the 4th hour and increase thereon with time. Statistical significance is $\mathrm{P}<0.01$. (From Powanda and Chang 2003, with permission.)

\section{THIRD GENERATION: NANODIMENSION COMPLETE ARTIFICIAL RED BLOOD CELLS}

\section{The First Artificial Red Blood Cell}

As discussed in the previous sections, the basic principle of nanobiotechnology-based blood substitutes (Chang 1964, 1971, 1972) has been developed into clinically useful $\mathrm{PolyHb}$, conjugated $\mathrm{Hb}$ and $\mathrm{PolyHb}$ with antioxidant activities. We are now ready to continue with the earlier approach of a complete artificial rbc (Chang 1957, 1964, 1972) (Fig. 6). As discussed below, the low circulation time that was the single major obstacle to the practical realization of the original complete artificial $\mathrm{rbc}$ has now been partly solved.

\section{Nanodimension Lipid Membrane Hb Vesicles}

\section{Increase in Circulation Time}

Extensive research has been carried out by many groups using lipid membrane to encapsulate $\mathrm{Hb}$ into $200 \mathrm{~nm}$ lipid-encapsulated $\mathrm{Hb}$ (LEH) (Djordjevich and Miller 1980; Phillips et al. 1999; Tsuchida et al. 2006). 


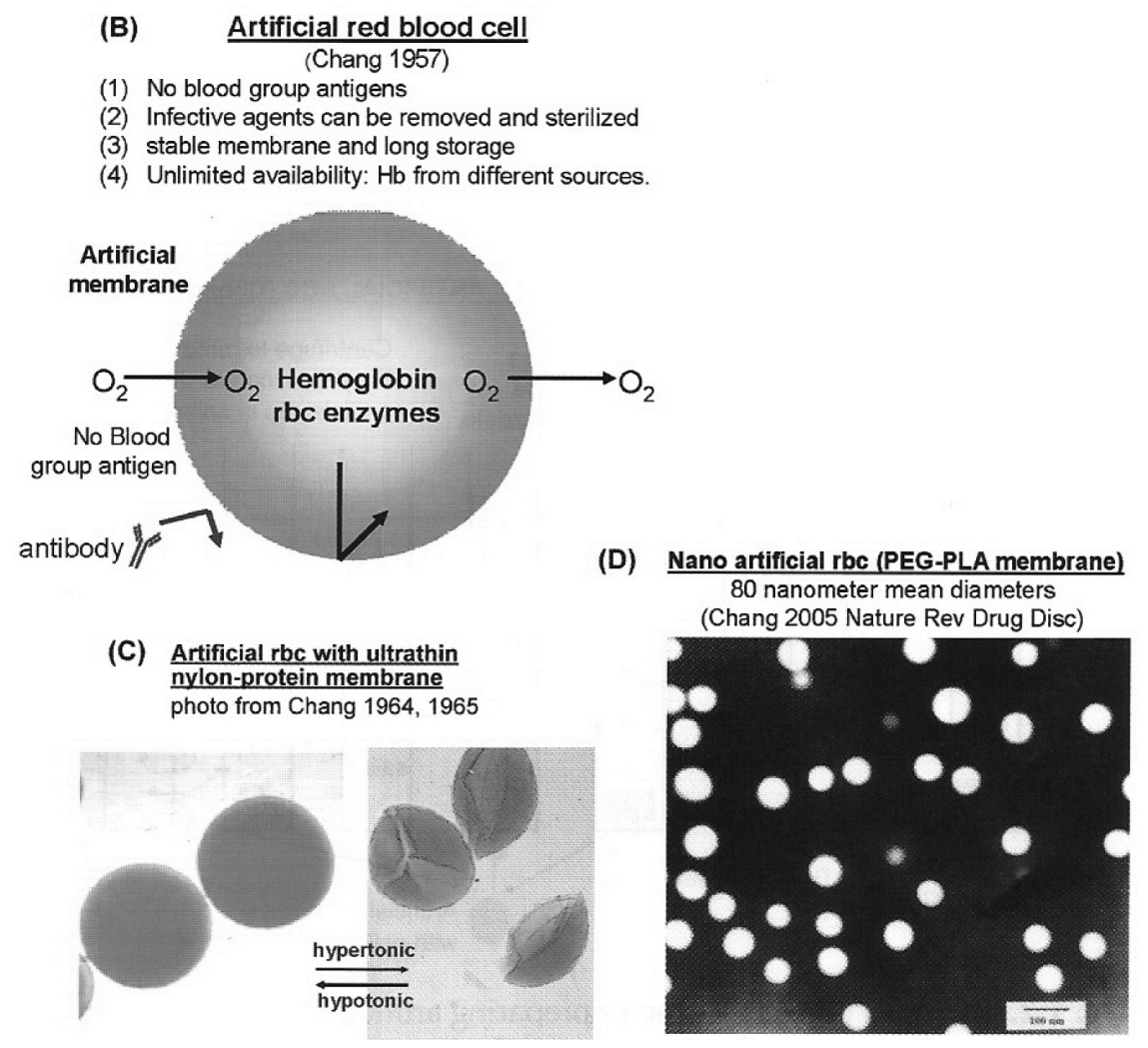

Fig. 6. Complete artificial red blood cells. (B) Schematic representation of artificial red blood cells. (C) Original micron dimension artificial red blood cell and (D) third generation nanodimension artificial red blood cell with $80 \mathrm{~nm}$ mean diameter. (From Chang 2007, with permission.)

Color image of this figure appears in the color plate section at the end of the book.

The use of PEG-lipid membrane to form $200 \mathrm{~nm}$ diameter PEG-lipid membrane artificial rbc increased the circulation time to a clinically useful level (Phillips et al. 1999). This allowed the development of LEH for animal studies. Results showed that these were effective in replacing loss of blood in hemorrhage shock and in exchange transfusion (Tsuchida et al. 2006). This is another important and major step in the development towards a more complete artificial rbc for clinical use.

\section{Further Developments}

Author: Is this change all right? We need a

LEH is useful for some clinical applications or other applications complete sentence here.

two further developments are needed: (1) Thamoun in tin 
must be entrolled. The smaller the particle, the larger will be the total surface area in the same volume of suspension. Thus, the total amount of lipid would be 10 times as high for the $1 \mathrm{ml}$ suspension of $200 \mathrm{~nm}$ lipid membrane artificial rbc than for rbc. A large amount of lipid can decrease the phagocytic function of the reticuloendothelial system (RES). In traumatic hemorrhagic shock, the RES needs to be efficient in removing contaminating microorganisms. Furthermore, in ischemic reperfusion, lipid may induce lipid peroxidation. (2) The other problem with the use of $\mathrm{LEH}$ is the oxidation of $\mathrm{Hb}$ into MetHb that cannot carry oxygen (Tsuchida et al. 2006). Reducing agents present in the circulating blood cannot cross the lipid membrane to reduce the rate of MetHb formation. Glucose that is needed for the MetHb reductase system inside rbc also cannot cross the lipid membrane of LEH.

\section{Nanodimension Biodegradable Polymeric Membrane Artificial rbc (Nano Artificial rbc)}

The next step is to markedly reduce the amount of lipid needed for the membrane and replace it with biodegradable polymeric membrane. We used our earlier background in biodegradable membrane artificial cells (Chang 1972) to prepare nanodimension artificial rbc (nano $\mathrm{rbc}$ ) of 80 nm mean diameter (Fig. 6) using PLA, PEG-PLA membrane and other biodegradable polymers (Chang 1997, 2005, 2007, 2009, 2010a, 2010c; Chang et al. 2003). The membrane thickness is $5-15 \mathrm{~nm}$. Since polymer is stronger than lipid and is also porous, much less membrane material is required. Thus, we can replace most of the $6 \mathrm{~g} / \mathrm{dl}$ of lipid membrane in LEH with a smaller amount of biodegradable polymeric membrane material (Fig. 7). This marked decrease in the lipid component would lessen effects on the RES and lessen lipid peroxidation in ischemia-reperfusion. Furthermore, polylactic acid (PLA) is degraded in the body into lactic acid, then to carbon dioxide (Fig. 7). These are all normal body metabolites. For a 500 $\mathrm{ml}$ suspension of PLA nano artificial $\mathrm{rbc}$, the total lactic acid produced is $83 \mathrm{mEq}$. The normal resting body lactic acid production is 1000-1400 $\mathrm{mEq} /$ day and the maximal body capacity to breakdown lactic acid is 7080 $\mathrm{mEq} /$ day. Thus, even in ischemic conditions, the amount of lactic acid produced can be well handled by the body.

\section{Characteristics of Nano Artificial rbc Including MetHb Reduction}

We can vary the $\mathrm{Hb}$ content in the PLA nano artificial rbc suspension from $3 \mathrm{~g} / \mathrm{dl}$ to $15 \mathrm{~g} / \mathrm{dl}$ (same as whole blood) (Fig. 7). This has normal $\mathrm{P}_{50}$, Hill's coefficient and Bohr coefficients. Red blood cell enzymes such as carbonic anhydrase, CAT, SOD and the MetHb reductase system have been enclosed within these nano artificial rbc and retain their activities (Chang 1979, 2005, 
92 Nanomedicine and the Cardiovascular System

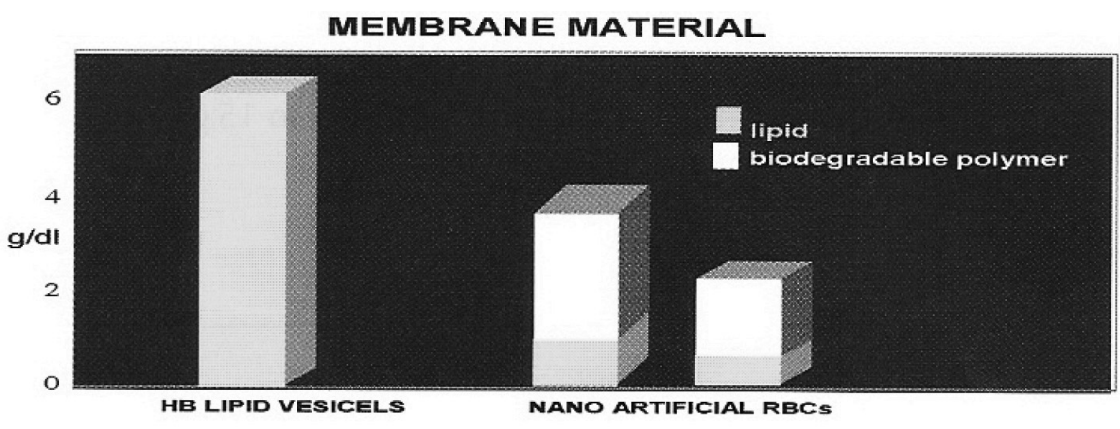

FATE OF POLYLACTIDE MEMBRANE
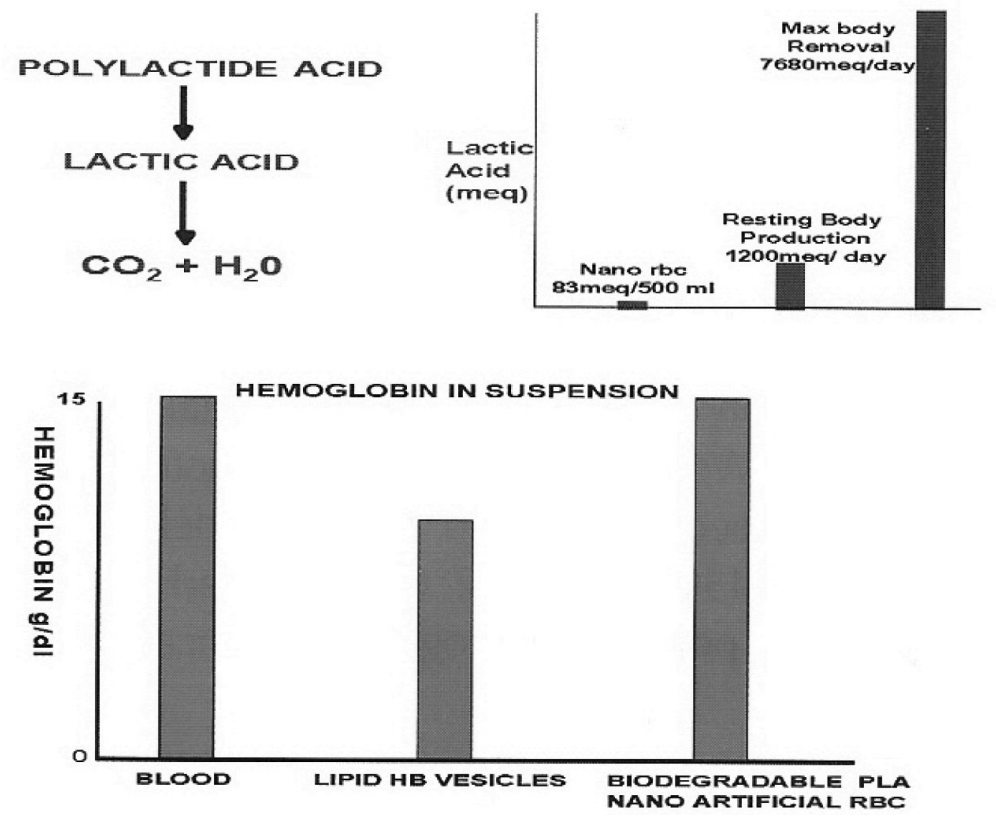

Fig. 7. Properties of nanodimension artificial red blood cells. Top: Amount of membrane material in hemoglobin lipid vesicles compared to polylactide membrane nano artificial red blood cells. Middle: Fate of polylactide membrane in PLA nano rbc compare to PLA metabolism in human. Lowe: Hb concentration reported. (From Chang 2007, with permission.)

2007; Chang et al. 2003). Unlike lipid membrane, biodegradable polymeric membrane is permeable to glucose. Thus, the inclusion of $\mathrm{rbc}$ MetHb reductase system prevents MetHb formation even at $37^{\circ} \mathrm{C}$ and we can also convert $\mathrm{MetHb}$ to $\mathrm{Hb}$ at $37^{\circ} \mathrm{C}$. Unlike lipid membrane, the nanocapsule 
membrane also allows plasma-reducing factors to enter the nanocapsules to prevent MetHb formation (Chang 2007).

\section{Circulation Time and In Vivo Properties}

In order to increase the circulation time, we synthesized a new PEG-PLA copolymer for the nano rbc membrane (Chang, 2005, 2007; Chang et al. 2003). After extensive research, we now have a circulation time in rats that is double that of glutaraldehye-crosslinked PolyHb. Since the RES in rat is much more efficient in removing particulate matter than in human, it is likely that the half life would be even longer in human. Nano artificial rbc did not have adverse effects on the histology and function of liver, spleen and kidney in rats when analyzed on days 1, 7 and 21 after infusions (Liu and Chang 2008).

\section{Oxygen Carrier with Platelet-like Activity}

In extensive blood loss, replacement of plasma volume and oxygen carrier is not enough. There is also the need to replace platelets and coagulation factors. We therefore use nanobiotechnology to develop a blood substitute that is an oxygen carrier with platelet-like properties.

$\mathrm{Hb}$ is crosslinked with fibrinogen to form a soluble nanobiotechnoligcal complex of PolyHb-fibrinogen (PolyHb-Fg) (Wong and Chang 2007). Exchange transfusion of $80 \%$ in rats of up to $98 \%$ using PolyHb-Fg did not result in clotting problems. The use of PolyHb alone resulted in some clotting problems after $80 \%$ exchange, worsening with further increase in blood replaced.

\section{PRESENT STATUS AND FUTURE}

Regional differences and the potentials of unknown infective agents must be included in any discussion of the prospect of blood substitutes and also in the degree of regulatory requirements (Chang 2010b). Blood substitute is urgently needed in regions of the world where there is severe shortage of donor blood because of cultural or religious beliefs that make people less willing to donate blood. It is also urgently needed in regions with higher incidence of infective agents such as HIV and thus higher potential for contaminated donor blood. It is less urgent in regions with lower incidence of HIV and where costly tests are being used to screen out infective agents in donated blood. On the other hand, it is also important to remember the past unexpected outbreak of HIV and hepatitis $C$ and the resulting contamination of donated blood that persisted for years until proper screening tests were developed. If this should happen again with 
yet unknown agents (e.g., avian flu, bioterrorism) then it would again be disastrous if no blood substitutes, even first-generation blood substitutes, were immediately available. Past experience has shown that it takes many years to develop ideas on blood substitutes into products and that lack of basic information has resulted in much failure and delays. It is important to carry out basic research to gain important basic information needed for the simultaneous development of blood substitutes. Meanwhile, new generations of modified $\mathrm{Hb}$ are being developed that can modulate the effects of nitric oxide for those clinical applications that may have potential problems related to oxygen radicals. A further development is the use of PEG-lipids or PEG-biodegradable polymer membranes to prepare nanodimension artificial rbc containing $\mathrm{Hb}$ and complex enzyme systems.

\section{APPLICATIONS TO AREAS OF HEALTH AND DISEASE}

Blood substitutes have important implications in transfusion medicine. Blood substitutes do not have blood group antigens. They can be used immediately on the spot without the need for matching and typing. Red blood cell membrane contains blood group antigens and donor blood requires typing and matching before it can be transfused into patients. This results in delays in emergency situations. Some blood substitutes could be stored for more than a year at room temperature. Donor blood requires storage at $4^{\circ} \mathrm{C}$ using standard method and is good for less than 42 d. Infective agents such as HIV and other viruses and microorganisms can be removed from blood substitutes. Red blood cells cannot be sterilized to remove hepatitis viruses, HIV and other potential emerging infective agents. Blood substitutes can be prepared using hemoglobin from different sources, whereas donor blood depends on human donors and may be in short supply especially in emergencies, war or disasters.

\section{Key Facts}

- The author started research on artificial red blood cells in 1957.

- However, there was no initial interest in this area of research until the 1980s, when HIV resulted in contaminated donor blood.

- Many patients were infected with contaminated blood. Unfortunately, there was no blood substitute to replace the HIV-contaminated donor blood.

- The spread of HIV stimulated industries to produce blood substitutes, but this was not as simple as people thought. In the 30 years since that time, no blood substitute has yet been approved for routine patient use in North America. 
- It is clear from that experience that blood substitutes are complex and cannot be developed into a product without more basic knowledge.

- The history of HIV contamination also suggests that we should intensify efforts to carry out both basic research and development in blood substitutes. We would not want to face the same problem of contaminated blood from another yet unknown epidemic. Suitable blood substitutes are also needed in some major surgeries and in cases of major disasters or war.

\section{Summary Points}

- Nanobiotechnology is the assembling of biological molecules into structures of 1 to $100 \mathrm{~nm}$.

- First-generation blood substitutes have been developed. These are socalled polyhemoglobin (PolyHb) formed by the nanobiotechnological assembling of hemoglobin molecules into soluble complexes.

- Unlike red blood cells, PolyHb is only an oxygen carrier.

- Unlike red blood cells, it does not have blood group antigens and can be used immediately on the spot without the need for typing or crossmatching.

- PolyHb can be stored for more than one year at room temperature and can therefore be easily transported and stored. Donor blood has to be stored at $4^{\circ} \mathrm{C}$ and only for $42 \mathrm{~d}$.

- Infective agents can be sterilized and removed from blood substitutes and therefore avoid problems related to contaminated donor blood.

- Unlike donor blood, there is an unlimited supply of blood substitutes because, in addition to human $\mathrm{Hb}(\mathrm{Hb})$, bovine $\mathrm{Hb}$ and recombinant human $\mathrm{Hb}$ can be used.

- Two types of PolyHb have been developed and tested in clinical trials in patients. Although one of them has been approved for routine clinical use in patients in South Africa, where there is higher incidence of HIV, in North America and other countries there is a need for further risk/ benefit ratio analysis and narrowing of use in more specific clinical conditions.

- PolyHb circulates effectively with a half time of $24 \mathrm{~h}$ and is therefore good for short-term use such as surgery and in emergencies. There are ways to increase its length of function, including repeated infusions and combination with erythropoietin.

- PolyHb is only an oxygen carrier and therefore cannot be used in clinical conditions with potential for ischemia-reperfusion injuries, as in sustained severe hemorrhagic shock, stroke or coronary ischemic diseases. 
- Second-generation biotechnological blood substitutes are being developed to solve this problem. For example, hemoglobin can be assembled with antioxidant enzymes such as CAT and SOD to form a soluble complex of PolyHb-SOD-CAT. Animal studies show that this can prevent ischemia-reperfusion injuries.

- In the meantime, complete artificial red blood cells of nanodimensions are being developed. One type is based on a bilayer lipid membrane artificial red blood cell of $200 \mathrm{~nm}$. Another type is based on biodegradable membrane artificial red blood cell of 120-80 nm. These have much higher circulation time than PolyHb. They also contain all the enzyme systems normally present in red blood cells.

\section{Definitions}

Crosslinking: The use of chemical linkage to assemble hemoglobin molecules.

Ischemia-reperfusion injury: After severe lack of oxygen (ischemia), introduction of oxygen carriers (reperfusion) can result in the production of oxygen radicals and tissue damage.

Nanobiotechnology: The assembling of biological molecules into nanodimension structures of 1 to $100 \mathrm{~nm}$ in diameter, membrane thickness or diameter of nanotubules.

Polyhemoglobin: The assembling of hemoglobin molecules into nanodimension structures by crosslinking.

\section{ABBREVIATIONS}

$\begin{array}{lll}\mathrm{CAT} & : & \text { catalase } \\ \mathrm{Fg} & : & \text { fibrinogen } \\ \mathrm{HIV} & : & \text { human immunodeficiency virus } \\ \mathrm{Hb} & : & \text { hemoglobin } \\ \mathrm{PolyHb} & : & \text { polyhemoglobin } \\ \mathrm{rbc} & : & \text { red blood cells } \\ \mathrm{SOD} & : & \text { superoxide dismutase }\end{array}$

\section{References}

Alayash, A.I., F. D'Agnillo and P.W. Buehler. 2007. First-generation blood substitutes: what have we learned? Biochemical and physiological perspectives. Expert Opin. Biol. Ther. 7: 665-675.

Atoji, T., M. Aihara, H. Sakai, E. Tsuchida and S. Takeoka. 2006. Hemoglobin vesicles containing methemoglobin and tyrosine to suppress methemoglobin formation in vitro and in vivo. Bioconjugate Chem. 17: 1241-1245. 
Buehler, P.W., C.R. Haney, A. Gulati, L. Ma and C.J. Hsia. 2004. Polynitroxyl hemoglobin: a pharmacokinetic study of covalently bound nitroxides to hemoglobin platforms. Free Radical Biol. Med. 37: 124-135.

Chang, E.J., T.H. Lee and K. Mun. 2004. Effects of polyhemoglobin-antioxidant enzyme complex on ischemia-reperfusion in kidney. Transplant Proc. 36: 1952-1954.

Chang, T.M.S. 1957. "Hemoglobin Corpuscles" Honours Physiology Research Report, 1957, Medical Library, McGill University.

Chang, T.M.S. 1964. Semipermeable microcapsules. Science 146: 524.

Chang, T.M.S. 1971. Stabilisation of enzymes by microencapsulation with aconcentrated protein solution or by microencapsulation followed by cross-linking with glutaraldehyde. Biochem. Biophys. Res. Common. 44: 1531-1536.

Chang, T.M.S. 1972. Artificial cells. Monograph. Charles C Thomas, Springfield, IL (full text available with free access at www.artcell.mcgill.ca).

Chang, T.M.S. 1976. Biodegradable semipermeable microcapsules containing enzymes, hormones,vaccines, and other biologicals. J Bioeng. 1: 25-32.

Chang, T.M.S. 1997. Monograph. Red blood cell substitutes: Principles, Methods, Products and Clinical Trials Vol I Karger/Landes Systems, Basel, Switzerland (free access on www.artcell.mcgill.ca).

Chang, T.M.S., D. Powanda and W.P. Yu. 2003. Ultrathin polyethylene-glycol-polylactide copolymer membrane nanocapsules containing polymerized $\mathrm{Hb}$ and enzymes as nanodimension RBC substitutes. Artificial Cells, Blood Substitutes and Biotechnology 31: 231-248.

Chang, T.M.S. 2005. Therapeutic applications of polymeric artificial cells. Nature Review: Drug Discovery 4: 221-235.

Chang, T.M.S. 2007. Monograph on "ARTIFICIAL CELLS: Biotechnology, nanotechnology, blood substitutes, regenerative medicine, bioencapsulation, cell/stem cell therapy" Singapore, World Science Publisher/Imperial College Press, pp. 1-452 (full text available with free access at www.artcell.mcgill.ca).

Chang, T.M.S. 2009. Nanobiotechnology for hemoglobin based blood substitutes. Critical Care Clinics 25: 373-382.

Chang, T.M.S. 2010c. Artificial Cells, Blood Substitutes and Nanomedicine. McGill University website www.artcell.mcgill.ca

Chang, T.M.S. 2010a. Blood replacement with engineered hemoglobin and hemoglobin nanocapsules. WIRE Nanomed. Nanobiotechnol. 2: 418-430.

Chang, T.M.S. 2010b. Editorial: Blood substitutes in 2010 Artificiall Cells, Blood Substitutes and Biotechnology 38: 1.

D'Agnillo, F., and T.M.S. Chang. 1998. PolyHb-superoxide dismutase. catalase as a blood substitute with antioxidant properties. Nature Biotechnol. 16: 667-671.

Djordjevich, L., and I.F. Miller. 1980. Synthetic erythrocytes from lipid encapsulated hemoglobin. Exp. Hematol. 8: 584.

Gould, S.A., et al. 2002. The life-sustaining capacity of human polymerized $\mathrm{Hb}$ when red cells might be unavailable. J. Am. Coll. Surg. 195: 445-452.

Jahr, J.S., C. Mackenzie, L.B. Pearce, A. Pitman and A.G. Greenburg. 2008. HBOC-201 as an alternative to blood transfusion: efficacy and safety evaluation in a multicenter phase III trial in elective orthopaedic surgery. J Trauma 64: 1484-1497.

Liu, Z.C., and T.M.S. Chang. 2008. Long term effects on the histology and function of livers and spleens in rats after 33\% toploading of PEG-PLA-nano artificial red blood cells. Artificial Cells, Blood Substitutes and Biotechnology 36: 513-524.

Moore, E.E., F.A. Moore, T.C. Fabian, A.C. Bernard, G.J. Fulda, D.B. Hoyt, T.M. Duane, L.J. Weireter Jr., G.A. Gomez, M.D. Cipolle, G.H. Rodman Jr., M.A. Malangoni, G.A. Hides, L.A. Omert and S.A. Gould. 2009. Human polymerized hemoglobin for the treatment of hemorrhagic shock when blood is unavailable: The USA multicenter trial. J. Am. Coll. Surg. 208: 1-13. 
98 Nanomedicine and the Cardiovascular System

Natanson, C., S.J. Kern, P. Lurie, S.M. Banks and S.M. Wolfe. 2008. Meta-analysis: risk of myocardial infarction and death: cell-free hemoglobin-based blood substitutes. JAMA 299: 2304-2312.

Olofsson, C., A. Torbjo, T. Johansson, S. Larsson, P. Nellga, S. Ponzer, B. Fagrell, R. Przybelski, P. Keipert, N. Winslow and R.M. Winslow. 2006. A multicenter clinical study of the safety and activity of maleimide-polyethylene glycol-modified hemoglobin (Hemospan ${ }^{\circledR}$ ) in patients undergoing major orthopedic surgery. Anesthesiology 105: 1153-1163.

Perutz, M.F. 1989. Myoglobin and Hb: Role of distal residues in reactions with haem ligands. Trends Biochem. Sci. 14: 42-44.

Phillips, W.T., R.W. Klipper, V.D. Awasthi, A.S. Rudolph, R. Cliff, V. Kwasiborski and B.A. Goins. 1999. Polyethylene glyco-modified liposome-encapsulated hemoglobin: a long circulating red cell substitute. J. Pharm. Exp. Ther. 288: 665-670.

Powanda, D. and T.M.S. Chang. 2002. Cross-linked polyHb-superoxide dismutase-catalase supplies oxygen without causing blood brain barrier disruption or brain edema in a rat model of transient global brain ischemia-reperfusion Artificial Cells, Blood Substitutes and Biotechnology 30: 25-42.

Tsuchida, E., H. Sakai, H. Horinouchi and K. Kobayashi. 2006. Hemoglobin-vesicles as a transfusion alternative. Artificial Cells, Blood Substitutes and Biotechnology 34: 581-588.

Winslow, R.M. (ed.). 2006. Blood Substitutes. Academic Press, Amsterdam. $\leftarrow$

Wong, N., and T.M.S. Chang. 2007. Polyhemoglobin-fibrinogen: a novel blood substitutes with platelet-like activity for extreme hemodilution. Artificial Cells, Blood Substitutes and Biotechnology 35: 481-489.

Yu, B.L., Z.C. Liu and T.M.S. Chang. 2006. PolyHb with different percentage of tetrameric $\mathrm{Hb}$ and effects on vasoactivity and electrocardiogram. Artificial Cells, Blood Substitutes and Biotechnology 34: 159-175.

Yu, B., M.J. Raher, G.P. Volpato, K.D. Bloch, F. Ichinose and W.M. Zapol. 2008. Inhaled nitric oxide enables artificial blood transfusion without hypertension Circulation 15(117): 1982-1990.

Yu, B., M. Shahid, E.M. Egorina, M.A. Sovershaev, M.J. Raher, C. Lei, M.X. Wu, K.D. Bloch and W.M. Zapol. 2010. Endothelial dysfunction enhances vasoconstriction due to scavenging of nitric oxide by a hemoglobin-based oxygen carrier. Anesthesiology 112: 586-594. 